\title{
Estimativas do albedo em três ecossistemas da floresta amazônica
}

\author{
Mário de M. V. B. R. Leitão ${ }^{1}$, Jesus M. dos Santos² \& Gertrudes M. de Oliveira ${ }^{3}$ \\ 1 DCA/CCT/UFCG. CP 10099, Av. Aprígio Veloso, 882, CEP 58109-970, Campina Grande, PB. Telefax: (83) $310-1202$. \\ E-mail: miranda@dca.ufpb.br (Foto) \\ 2 INPE. São José dos Campos, SP. Fone: (12) 3945-6010. E-mail: marden@inpe.br \\ ${ }^{3}$ Doutoranda, CDRN/CCT/UFCG. Fone: (83) 310-1199. E-mail: gertrude@dca.ufpb.br
}

Protocolo $084-7 / 6 / 2001$

\begin{abstract}
Resumo: Os albedos relativos às radiações de ondas curtas, infravermelho e fotossinteticamente ativa foram estimados para três ecossistemas da floresta amazônica. A pesquisa foi conduzida nas áreas de Campina e Campinarana, na Reserva Biológica de Campina, localizada no quilômetro 46 da rodovia BR-174 (2 $\left.34^{\prime} \mathrm{S} ; 60^{\circ} 02^{\prime} \mathrm{W}\right)$, que liga Manaus a Caracaraí e na área de Mata Densa, na Reserva Florestal Ducke ( $2^{\circ} 57^{\prime} \mathrm{S}$; $59^{\circ} 57^{\prime} \mathrm{W}$ ). Para obtenção dos dados nos diferentes tipos de vegetação, foi instalado, numa torre metálica, um conjunto de sensores para medir radiações de ondas curtas, infravermelho e fotossinteticamente ativa, incidentes e refletidas sobre os dosséis vegetativos, além de sensores para medir radiações de ondas curtas incidente, de ondas curtas refletida e fotossinteticamente ativa, próximo à superfície do solo dos dosséis. Para a aquisição dos dados foram utilizados quatro microloggers, os quais foram programados para efetuarem, no período das 5 h 51 min às 18 h, leituras a cada segundo e médias a cada minuto. Os resultados mostraram que os albedos de ondas curtas, infravermelho e radiação fotossinteticamente ativa (PAR), nos três tipos de vegetação, podem ser estimados em função do ângulo de elevação do sol, com boa precisão para qualquer instante do dia.
\end{abstract}

Palavras-chave: Mata Densa, Campina, Campinarana, reflexão

\section{Estimation of albedo in three ecosystems of the Amazon forest}

\begin{abstract}
The albedos of shortwave, infrared and photosynthetically active radiations (PAR) were estimated for three ecosystems of the Amazon forest. The research was conducted in the

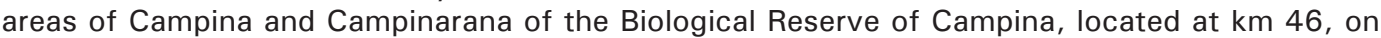
Highway BR-174 which connects Manaus and Caracarai $\left(2^{\circ} 34^{\prime} \mathrm{S} ; 60^{\circ} 2^{\prime} \mathrm{W}\right)$ and in the area of "Mata Densa" in the Ducke Forest Reserve (2 ${ }^{\circ} 57^{\prime} \mathrm{S}$; $\left.59^{\circ} 57^{\prime} \mathrm{W}\right)$. A metallic tower, with set of sensors for measuring the incident and canopy reflected shortwave, infrared and photosynthetically active radiations was installed over each of the above mentioned canopies. Similar sensors were also installed at the soil surface to measure the cited parameters. Four microloggers were used for acquiring the daily data from $5 \mathrm{~h} 51 \mathrm{~min}$ to $18 \mathrm{~h}$ at intervals of one second and the average values for each minute were obtained and stored. The results show that the albedos of the shortwave, infrared and photosynthetically active radiations for the three canopies can be estimated, as a function of the solar elevation angle, with good precision for anytime of the day.
\end{abstract}

Key words: Mata Densa, Campina, Campinarana, reflection

\section{INTRODUÇÃO}

O albedo é um parâmetro muito importante no balanço de radiação de uma superfície e bastante utilizado em modelos climáticos e agrometeorológicos, tais como estimativas do fluxo de vapor d'água e do saldo de radiação, dentre outros; entretanto, este parâmetro nem sempre está disponível, visto que sua medida não é efetuada de forma rotineira, como ocorre com muitos outros parâmetros meteorológicos. Na Amazônia brasileira, exceto a pesquisa realizada por Leitão (1994) nos ecossistemas de Campina, Campinarana e Mata Densa, os dados disponíveis de albedo se referem apenas a este último ecossistema, uma floresta muito diferente das duas primeiras em termos de altura, abertura e estrutura de dossel vegetativo. Essa escassez de dados na Amazônia ocorre devido, principalmente, às dificuldades operacionais e aos custos extremamente elevados dos equipamentos necessários à realização das pesquisas. $\mathrm{O}$ albedo de uma superfície vegetada varia com o ângulo de elevação do sol, tipo de vegetação, condições de umidade do ar e da superfície, umidade e tipo de 
solo, além da quantidade e do tipo de nuvens (Blad \& Baker, 1972; Leitão, 1989; Azevedo et al., 1990). No caso de vegetação de floresta, como a folhagem é agrupada na copa, com picos e depressões organizados nas superfícies dos dosséis, grande quantidade de radiação solar incidente penetra antes de ser refletida (Shuttleworth et al., 1984). Segundo Eck \& Deering (1992) isso resulta numa acentuada captura de radiação solar e, conseqüentemente, numa baixa reflexão. Conforme Pereira (1997) menos de $10 \%$ da radiação solar incidente atinge o piso da floresta. Durante o dia, o albedo decresce com a elevação do sol alcançando, em geral, valores máximos próximo ao nascer e pôr-do-sol, enquanto os valores mínimos são observados em torno do meio-dia (Rijks, 1967; Stewart, 1971; Viswanadham, 1972; Pinker, 1982; Leitão, 1989; Gash \& Shuttlewort, 1991). Eck \& Deering (1992) concluíram que o albedo menor para ângulos de elevação solar maiores se deve a maior penetração de radiação no dossel vegetativo, resultando no aumento da absorção e espalhamento da radiação. Quando o ângulo de elevação solar é pequeno, a radiação incidente não penetra no interior do dossel, ocorrendo redução da absorção por espalhamento múltiplo, o que concorre para uma reflexão maior da superfície e, em conseqüência, para um albedo maior.

As dificuldades em se obter medidas reais de radiação e, conseqüentemente, do albedo, na floresta amazônica, têm levado muitos pesquisadores a usarem, em geral, em modelos climáticos, um valor único e constante do albedo para toda a área vegetada da Amazônia o que, na prática, não reflete a realidade, pois a floresta amazônica é constituída de vários ecossistemas, com dosséis muito diferentes (Leitão, 1994). Diante do exposto objetivou-se, com este trabalho, analisar medidas do albedo obtidas nos ecossistemas de Campina, Campinarana e Mata Densa na floresta amazônica, e propor estimativas de albedo para três faixas de comprimento de onda de radiação solar: ondas curtas, infravermelho e fotossinteticamente ativa.

\section{MATERIAL E MÉTODOS}

\section{Experimento de campo}

Os dados utilizados neste trabalho foram obtidos em duas campanhas experimentais, realizadas de $1 / 9$ a 30/9/91 e de 23/8 a 30/9/93. A pesquisa foi conduzida em três áreas da floresta amazônica: Campina e Campinarana, ambas situadas na Reserva Biológica de Campina, localizada na altura do quilômetro 46 da rodovia BR-174 ( $\left.2^{\circ} 34^{\prime} \mathrm{S} ; 60^{\circ} 2^{\prime} \mathrm{W}\right)$, que liga Manaus a Caracarai e na área de Mata Densa, na Reserva Ducke, km 23 da rodovia Manaus-Itacoatiara $\left(2^{\circ} 57^{\prime} \mathrm{S} ; 59^{\circ} 57^{\prime} \mathrm{W}\right)$ conforme indica a Figura 1. Na primeira campanha experimental, o período de observações em cada área foi de $6 \mathrm{~d}$, enquanto na segunda foi de $8 \mathrm{~d}$. Para obtenção dos dados sobre os dosséis vegetativos, foi erguida uma torre metálica de $10 \mathrm{~m}$ de altura na área de Campina, e outra de $17 \mathrm{~m}$, na Campinarana; na Mata Densa utilizou-se uma torre de $45 \mathrm{~m}$, já existente. Para determinação da radiação global incidente e refletida, bem como, da radiação infravermelha incidente, foram utilizados piranômetros Eppley PSP, cuja sensibilidade é de $9 \mu \mathrm{V} \mathrm{W}^{-1} \mathrm{~m}^{-2}$; para determinação da radiação infravermelha refletida, piranômetros LI-COR 200SZ com sensibilidade de $8 \mu \mathrm{V} \mathrm{W}^{-1} \mathrm{~m}^{-2}$; e para determinação da radiação líquida, saldo radiômetro com sensibilidade de $5 \mu \mathrm{V} \mathrm{W}^{-1} \mathrm{~m}^{-2}$. Os instrumentos foram instalados a $2,5 \mathrm{~m}$ acima de cada dossel vegetativo. Para medir a radiação global incidente e radiação refletida no interior dos dosséis, foram instalados, a $0,5 \mathrm{~m}$ acima da superfície do solo, radiômetros de célula de silício com sensibilidade de $20 \mu \mathrm{V} \mathrm{W}^{-1} \mathrm{~m}^{-2}$; 16 radiômetros para medir radiação global incidente e 16 radiômetros para medir radiação refletida pela superfície, os quais foram montados aos pares, de modo que cada suporte tivesse um sensor na parte superior (radiação incidente) e outro na parte inferior (radiação refletida). Também, foram instalados 16 piranômetros LI-COR 200SZ, para medir a radiação fotossinteticamente ativa incidente na superfície do solo. Desse modo, os dados foram amostrados em 16 pontos, numa malha de $5 \times 5 \mathrm{~m}$, que ocupou uma área de $15 \times 15 \mathrm{~m}$. Para aquisição dos dados, quatro microloggers (2 Datalogger $21 \mathrm{X} \mathrm{e} 2$ Datalogger CR10) foram programados para, no período das $5 \mathrm{~h} 51 \mathrm{~min}$ às $18 \mathrm{~h}$, efetuarem leituras a cada segundo e médias a cada minuto.

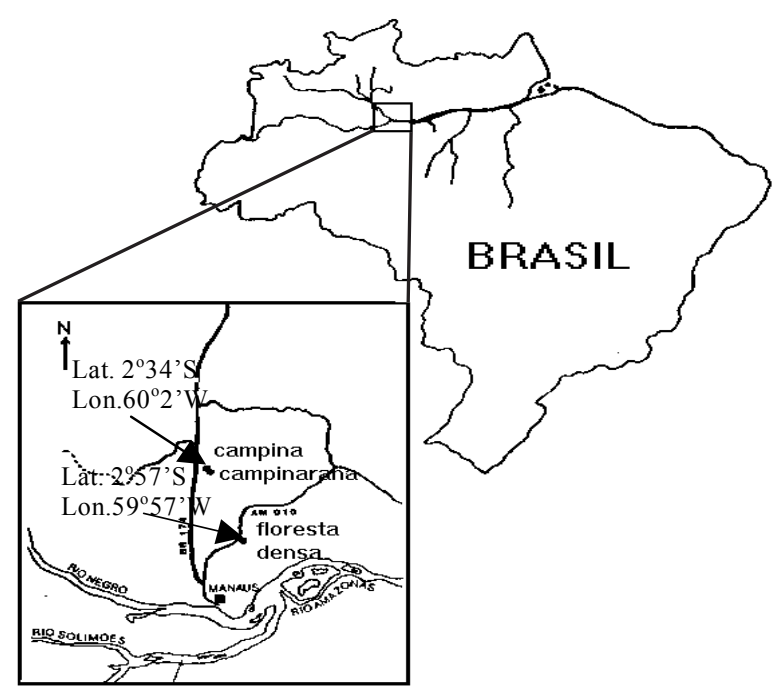

Figura 1. Localização das áreas experimentais

\section{Metodologia}

A radiação infravermelho incidente na base do dossel $\left(\mathrm{IR}_{\mathrm{b}} \downarrow\right)$ foi obtida pela diferença entre a radiação global $\left(K_{b} \downarrow\right)$ e a radiação fotossinteticamente ativa $\left(\mathrm{PAR}_{\mathrm{b}} \downarrow\right)$ incidentes na base, ou seja:

$$
\mathrm{IR}_{\mathrm{b}} \downarrow=\mathrm{K}_{\mathrm{b}} \downarrow-\mathrm{PAR}_{\mathrm{b}} \downarrow
$$

em que todos os termos da Eq. (1) estão em $\mathrm{W} \mathrm{m}^{-2}$.

Como o albedo é a razão entre a radiação refletida e a radiação incidente, tomando-se como base as radiações nas faixas espectrais global $(0,3$ a $3,0 \mu \mathrm{m}), \operatorname{PAR}(0,4$ a $0,7 \mu \mathrm{m})$ e infravermelho próximo $(0,7$ a $3 \mu \mathrm{m})$ determinou-se o albedo para quatro situações: albedo de ondas curtas da superfície foliar $\left(\mathrm{r}_{\mathrm{c}}\right)$; albedo PAR $\left(\mathrm{r}_{\mathrm{p}}\right)$; albedo infravermelho próximo $\left(\mathrm{r}_{\mathrm{i}}\right)$ e albedo de ondas curtas da superfície do solo $\left(\mathrm{r}_{\mathrm{S}}\right)$. Para a obtenção do albedo instantâneo dividiu-se a radiação refletida pela radiação incidente observada a cada minuto durante o período diurno, enquanto o albedo médio diário foi determinado efetuando-se 
a razão entre os valores diários de radiação refletida e radiação incidente durante o período de observação. Deste modo, os cálculos foram efetuados utilizando-se as seguintes equações:

$$
\begin{gathered}
\mathrm{r}_{\mathrm{c}}=\frac{\mathrm{K} \uparrow}{\mathrm{K} \downarrow} \\
\mathrm{r}_{\mathrm{p}}=\frac{\mathrm{PAR} \uparrow}{\mathrm{PAR} \downarrow} \\
\mathrm{r}_{\mathrm{i}}=\frac{\mathrm{IR} \uparrow}{\mathrm{IR} \downarrow} \\
\mathrm{r}_{\mathrm{s}}=\frac{\mathrm{K}_{\mathrm{s}} \uparrow}{\mathrm{K}_{\mathrm{s}} \downarrow}
\end{gathered}
$$

em que: $\mathrm{K} \uparrow$ e $\mathrm{K} \downarrow$ são os fluxos de radiação de ondas curtas refletido e incidente na superfície foliar; PAR $\uparrow$ e PAR $\downarrow$ são os fluxos de radiação fotossinteticamente ativa refletido e incidente; IR $\uparrow$ e IR $\downarrow$ são os fluxos de radiação infravermelho próximo refletido e incidente; e $K_{s} \downarrow$ e $K_{s} \uparrow$ são os fluxos de radiação de ondas curtas incidente e refletido na base do dossel, respectivamente. Todos os termos de radiação são dados em $\mathrm{W} \mathrm{m}{ }^{-2}$. O albedo também foi modelado em função do ângulo de elevação do sol $(\alpha)$ tomando-se como base as equações obtidas por regressão; no caso de $r_{c}$ e $r_{i}$ usou-se:

$$
\mathrm{r}=\mathrm{c}_{1}+\mathrm{c}_{2} \alpha+\mathrm{c}_{3} \alpha^{2}
$$

em que: $c_{1}, c_{2}$ e c são coeficientes de regressão polinomial. E, no caso de $r_{p}$ a equação:

$$
\mathrm{r}=\mathrm{c}_{1}+\mathrm{c}_{2} \alpha
$$

Para determinação do ângulo $\alpha$, utilizou-se a equação:

$$
\operatorname{sen} \alpha=\operatorname{sen} \phi \operatorname{sen} \delta+\cos \phi \cos \delta \cos \eta
$$

em que: $\phi$ é a latitude do local; $\delta$ é a declinação do sol e $\eta$ é o ângulo horário. Os valores diários de $\delta$ foram calculados pela equação de Spencer (1971):

$$
\delta=\left(\begin{array}{l}
0,006918-0,399912 \cos \Gamma+ \\
0,070257 \operatorname{sen} \Gamma-0,006758 \cos 2 \Gamma+ \\
0,000907 \operatorname{sen} 2 \Gamma-0,002697 \cos 3 \Gamma+ \\
0,00148 \operatorname{sen} 3 \Gamma
\end{array}\right)\left(\frac{180}{\pi}\right)
$$

em que: $\Gamma=2 \pi(\mathrm{d}-1) / 365$, sendo $\mathrm{d}$ o dia do ano, conforme o calendário Juliano.

Para a utilização da Eq. (8), minuto a minuto do nascer ao pôr-do-sol, efetuou-se uma adaptação no termo cosๆ, que passou a ter a seguinte forma:

$$
\cos \eta=\cos (180-0,25 \mathrm{~m})
$$

em que m é o número de minutos transcorridos ao longo do dia.

\section{RESULTADOS E DISCUSSÃO}

Os albedos médios diários relativos às radiações de ondas curtas, infravermelho e PAR para os dosséis de Mata Densa, campinarana e campina são apresentados na Figura 2. Observa-se que o dossel de Mata Densa apresentou, para os três tipos de albedo, a maior variação ao longo do dia, com valores pouco

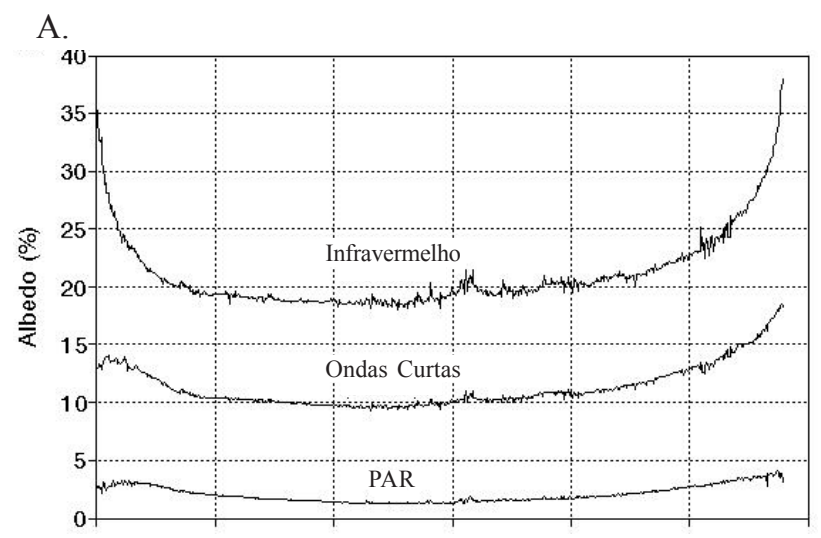

B.

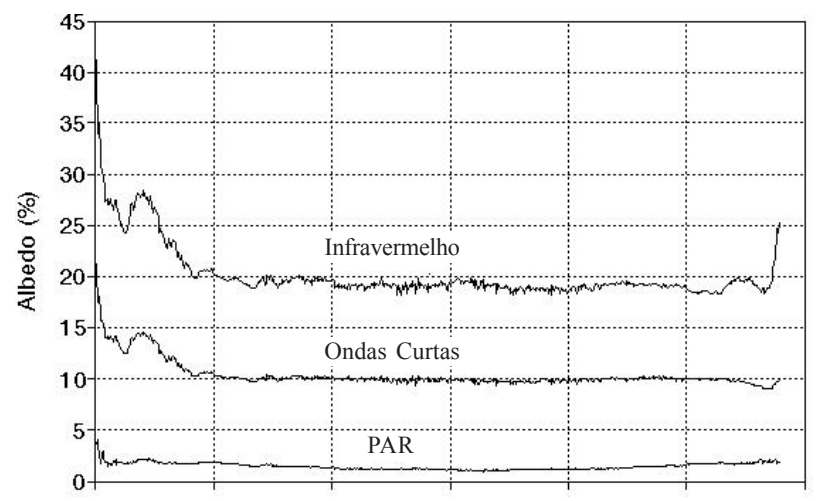

C.

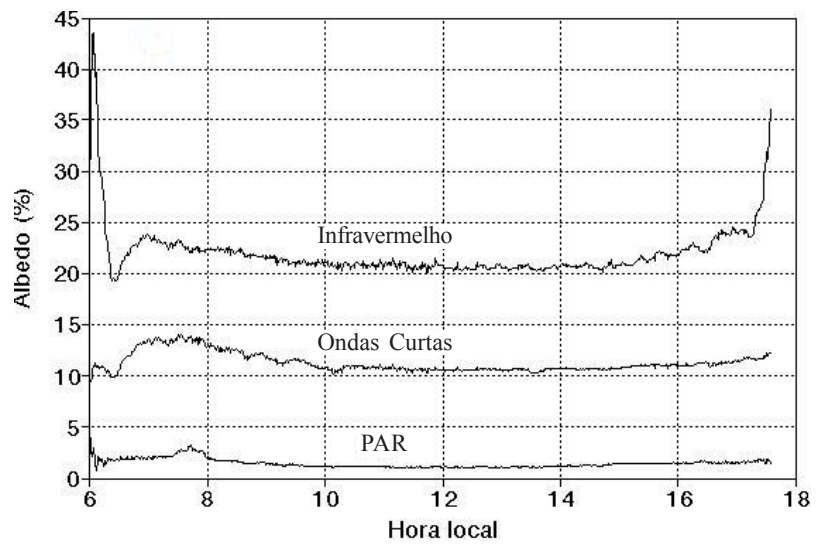

Figura 2. Albedo médio relativo às radiações de ondas curtas, infravermelho e PAR observado a cada minuto para os três ecossistemas: (A) Mata Densa; (B) Campinarana e(C) Campina, no período de setembro de 1993 
maiores à tarde, em relação ao período da manhã. Tal variação foi também comentada por Pereira (1997). Já na Campinarana (Figura 2B), os albedos variaram muito pouco ao longo do dia, exceto no período de 6 às $8 \mathrm{~h}$ e no final da tarde; no entanto, tais variações devem ser observadas com cautela, visto que próximo do nascer e pôr do sol, as irregularidades no topo dos dosséis podem produzir reflexões laterais aumentando o albedo, por isso, na maioria dos estudos não se considera o albedo próximo do nascer e pôr do sol. Na Campina (Figura 2C), da mesma forma que nos outros dois dosséis, também se observa variação acentuada dos albedos nas primeiras e últimas horas do dia. Esta variação tem sido observada em outras pesquisas (Proctor et al., 1972; Nkemdirim, 1973; Lomas et al., 1974) e resulta de sombreamento, devido a não homogeneidade das copas dos dosséis.

Observa-se, ainda que, para os três dosséis (Figura 2), o albedo relativo a radiação infravermelho apresentou a maior flutuação ao longo do dia, com destaque para o dossel de Mata Densa, que apresentou a maior flutuação; já o dossel de Campina mostrou a menor flutuação para os três tipos de albedo. Verifica-se também que o albedo da radiação PAR, principalmente nos dosséis de Campinarana e Campina, manteve-se praticamente constante ao longo do dia. Tais situações podem ser comprovadas analisando-se o desvio padrão do albedo para cada dossel mostrado na Tabela 1.

Tabela 1. Desvio-padrão do albedo diário

\begin{tabular}{lccc}
\hline \multirow{2}{*}{ Dossel } & \multicolumn{3}{c}{ Desvio-padrão do Albedo $\left(\mathrm{W} \mathrm{m}^{-2}\right)$} \\
\cline { 2 - 4 } & $\mathrm{r}_{\mathrm{c}}$ & $\mathrm{r}_{\mathrm{i}}$ & $\mathrm{r}_{\mathrm{p}}$ \\
\hline Mata Densa & 1,07 & 0,80 & 0,35 \\
Campinarana & 0,56 & 0,61 & 0,20 \\
Campina & 0,37 & 0,18 & 0,18 \\
\hline
\end{tabular}

$r_{c}$ - albedo de ondas curtas da superfície foliar; $r_{i}$ - albedo infravermelho próximo; $r_{p}$ - albedo PAR

Os três tipos de albedo modelados em função do ângulo de elevação solar a cada meia hora, entre $6 \mathrm{~h} 30 \mathrm{~min}$ e $17 \mathrm{~h} 30 \mathrm{~min}$, são apresentados na Figura 3. Observa-se que as equações obtidas para os albedos $r_{i}$ e $r_{c}$ são polinômios de $2^{\circ}$ grau, enquanto para $r_{p}$ são equações de $1^{\circ}$ grau. Verifica-se também que todos os coeficientes de determinação $\left(\mathrm{R}^{2}\right)$ estão acima de 0,91 e que o dossel de campina apresentou os melhores coeficientes $\left(\mathrm{R}^{2}=0,99\right)$ para os três tipos de albedo, indicando que as equações podem proporcionar boas estimativas.

Como as equações foram obtidas com base nos dados observados em 1993, para mostrar suas performances, estimou-se os albedos $r_{i}, r_{c}$ e $r_{p}$ para cada minuto dos dias 11,16 e 25/9/ 1991, a fim de compará-los aos valores observados nesses dias. Comparando-se os três tipos de albedo estimados em função do ângulo de elevação do sol aos observados (Fig. 4), verifica-se concordância muito boa das curvas do albedo estimado com as curvas do albedo observado; isto mostra que, para os três dosséis estudados, os albedos $r_{i}, r_{c}$ e $r_{p}$, podem ser estimados com razoável precisão para qualquer instante do dia.

Os valores médios diários de $r_{i}, r_{c}$ e $r_{p}$ para os três dosséis, são apresentados na Tabela 2, na qual se verifica que o albedo de ondas curtas da Campina foi igual ao da Mata Densa $(11,3 \%)$ e o da Campinarana menor que estes, apenas $0,9 \%$;
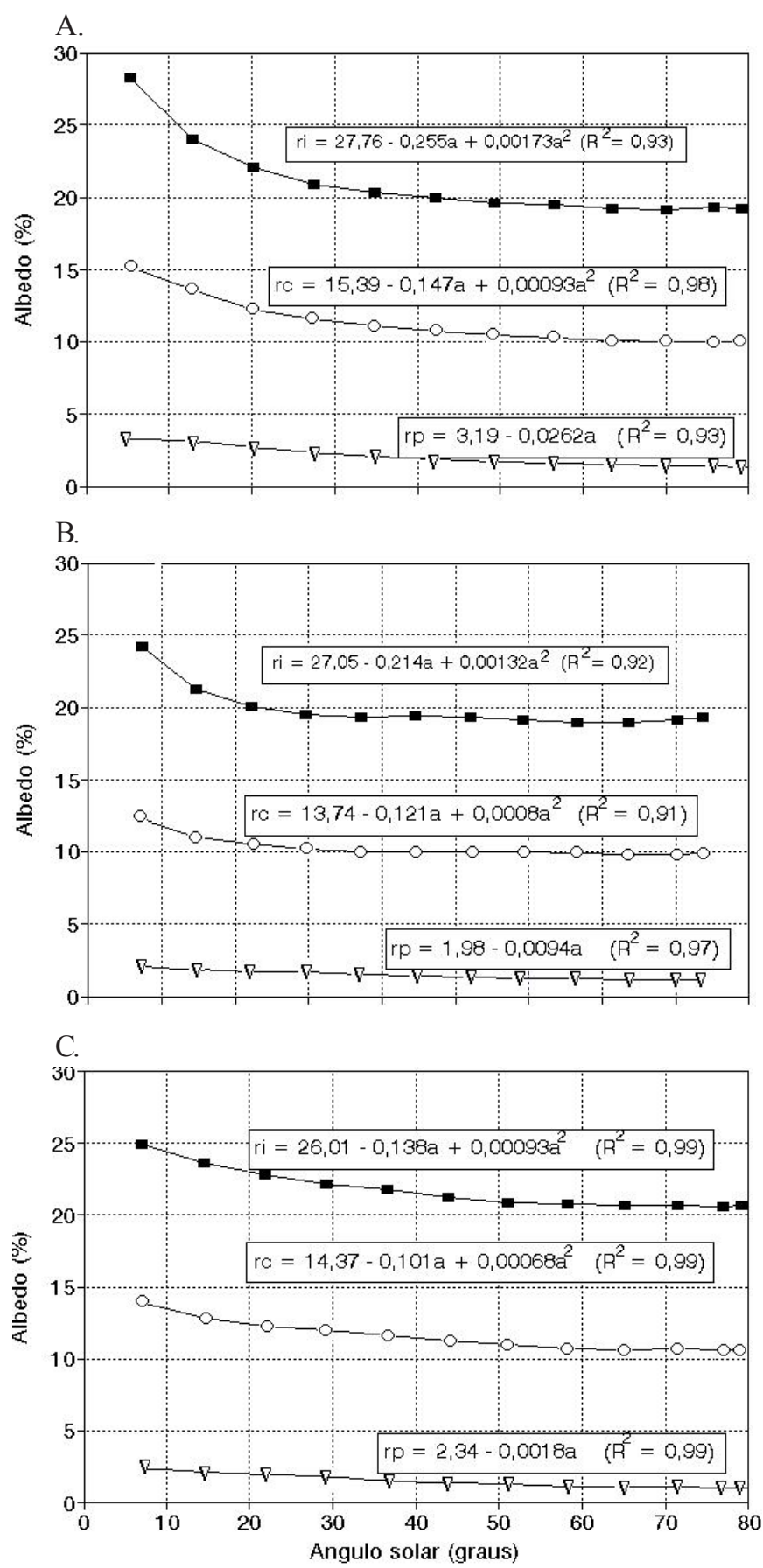

$\rightarrow$ Infravermelho $-\circ$ - Ondas Curtas $\nabla$ - PAR

Figura 3. Albedo relativo à radiação de ondas curtas, infravermelho e PAR, estimado em função do ângulo de elevação solar $(\alpha)$, a cada meia hora, entre $6 \mathrm{~h} 30 \mathrm{~min}$ e $17 \mathrm{~h} 30$ min para os três ecossistemas: (A) Mata Densa; (B) Campinarana e (C) Campina, no mês de setembro de 1993

Tabela 2. Albedo médio diário da copa dos três dosséis

\begin{tabular}{lccc}
\hline \multirow{2}{*}{ Dossel } & \multicolumn{3}{c}{ Tipo de Albedo (\%) } \\
\cline { 2 - 4 } & $\mathrm{r}_{\mathrm{c}}$ & $\mathrm{r}_{\mathrm{i}}$ & $\mathrm{r}_{\mathrm{p}}$ \\
\hline Mata Densa & 11,3 & 21,2 & 2,0 \\
Campinarana & 10,4 & 20,2 & 1,5 \\
Campina & 11,3 & 22,0 & 1,4 \\
\hline
\end{tabular}



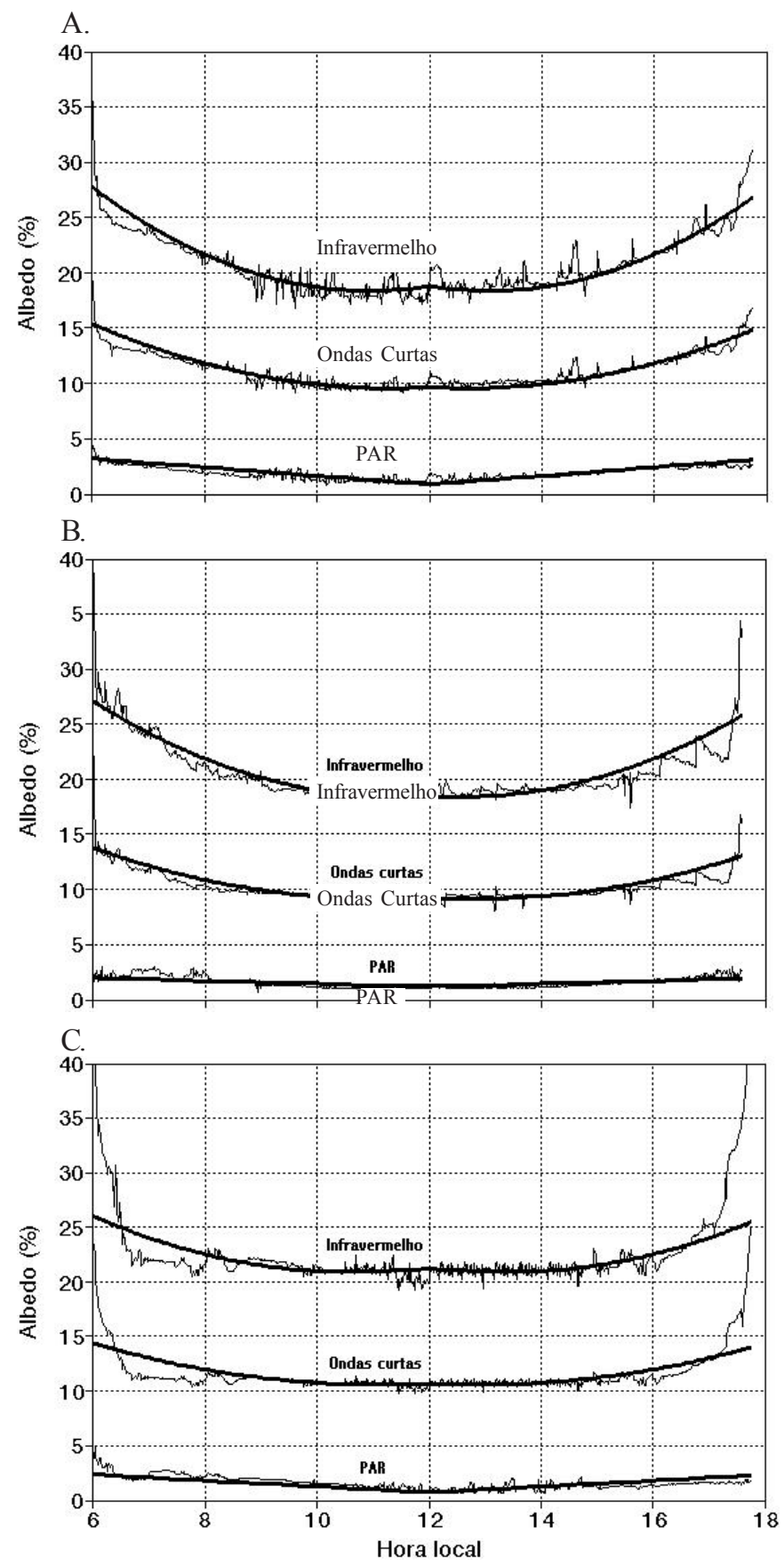

Worm Medido - Modelado

Figura 4. Albedos modelado e observado para os três ecossostemas: (A) Mata Densa (25/9/1991); (B) Campinarana (16/9/1991) e (C) Campina (11/9/1991)

Shuttlheworth et al. (1984) encontraram, para uma área de Mata Densa, um albedo médio diário anual de $12,5 \%$ para ondas curtas; já o albedo infravermelho da mata densa foi $1 \%$ maior que o da Campinarana e menor $0,8 \%$ que o da Campina, enquanto o albedo PAR apresentou-se praticamente igual na Campina e Campinarana, e cerca de $0,5 \%$ mais elevado na mata densa. Na Tabela 3 é apresentado o albedo médio diário de ondas curtas da superfície do solo das três áreas, o qual se mostra relativa-mente elevado, devido a quantidade de radiação direta incidente na base dos dosséis ser pequena e ocorrer por períodos de curta duração, principalmente no dossel mais fechado, ou seja, o de Mata Densa.

Tabela 3. Albedo médio diário do solo dos três dosséis

\begin{tabular}{lc}
\hline \multicolumn{1}{c}{ Dossel } & Albedo (\%) \\
\hline Mata Densa & 30,5 \\
Campinarana & 21,6 \\
Campina & 25,2 \\
\hline
\end{tabular}

\section{CONCLUSÕES}

1. Apesar das diferenças de densidade e altura de plantas dos dosséis vegetativos de Campina, Campinarana e Mata Densa, os albedos relativo as radiações de ondas curtas, infravermelho e fotossinteticamente ativa apresentaram pequenas variações. No entanto, em termos proporcionais, o albedo PAR nas áreas de Campina e Campinarana foi menor que o da área de Mata Densa, muito provavelmente por serem esses dosséis mais abertos e suas superfícies foliares terem tonalidade verde diferente daquela da Mata Densa.

2. O dossel de Campinarana, por apresentar uma superfície com tonalidade de verde mais escura, foi o dossel que absorveu mais radiação infravermelho e apresentou a menor reflexão dessa radiação.

3. Os albedos relativos às radiações de ondas curtas e infravermelho na Mata Densa, durante o período da tarde, apresentaram valores superiores aos observados pela manhã para o mesmo ângulo de elevação solar. Essa situação pode estar associada tanto a redução do teor de umidade nas folhas, que contribui para diminuir a absorção de radiação pela superfície foliar, como a redução da radiação direta e conseqüente incremento da radiação difusa devido ao aumento da nebulosidade a partir do início da tarde, algo comum e acentuado em regiões de floresta.

4. O albedo de ondas curtas mais elevado no solo da Mata Densa, pode ser um indicativo de que, em dosséis fechados, nas horas de pico o solo absorve mais radiação.

5. As comparações entre valores estimados e medidos, mostram que os albedos de ondas curtas, infravermelho e PAR, podem ser calculados em função do ângulo de elevação do sol, para qualquer instante do dia, com boa precisão.

\section{LITERATURA CITADA}

Azevedo, P.V.; Leitão, M.M.V.B.R.; Sousa, I.F.; Maciel, G.F. Balanço de radiação sobre culturas irrigadas no semi-árido do Nordeste do Brasil. Revista Brasileira de Meteorologia, Rio de Janeiro, v.5, n.1, p.403-410, 1990.

Blad, B.L.; Baker, D.G. Reflected radiation from a soybean crop. Agronomy Journal, Madison, v.64, p.277-280, 1972.

Eck, T.F.; Deering, D.W. Canopy albedo and transmittance in spruce-hemlock forest in mid-September. Agricultural and Forest Meteorology, Amsterdam, v.59, n.3-4, p.237-248, 1992. 
Gash, J.H.C.; Shuttleworth, W.J. Tropical deforestation: Albedo and the surface-energy balance. Climatic Change, Amsterdam, v.19, n.1-2, p.123-137, 1991.

Leitão, M. M.V.B.R. Balanço de radiação e energia numa cultura de soja irrigada. Campina Grande: DCA/CCT/UFPB, 1989. 111 p. Dissertação Mestrado

Leitão, M.M.V.B.R. Balanço de radiação em três ecossistemas da Floresta Amazônica: Campina, Campinarana e Mata Densa. São José dos Campos: INPE, 1994. 135p. Tese Doutorado

Lomas, J.; Schlesinger, E.; Lewin, J. Effects of environmental and crop factors on the evapotranspiration rate and water use efficiency of maize. Agricultural Meteorology, Amsterdam, v.13, p.239-251, 1974.

Nkemdirim, L.C. Ratiative flux relations over crops. Agricultural Meteorology, Amsterdam, v.11, p.229-242, 1973.

Pereira, A.R. Radiation regime of tropical rain forest. Revista Brasileira de Agrometeorologia, Santa Maria, v.5, n.2, p.1-8, 1997.

Pinker, R.T. The diurnal assymetry in the albedo of tropical forest vegetation. Forest Science, Amsterdam, v.28, p.297-234, 1982.
Proctor, J.T.A.; Kyle, J.W.; Davies, J.A. The radiation balance of the apple tree. Canadian Journal of Botany, Canadá, v.50, p.1731-1740, 1972.

Rijks, D.A. Water use by irrigated cotton in Sudan I: Reflection of short-wave radiation. Journal of Applied Ecology, London, v.4, p.561-568, 1967.

Shuttleworth, W.J; Gash, J.H.; Lloyd, C.R.; Moore, C.J.; Roberts, J.; Marques Filho, A.O.; Fisch, G.; Silva Filho, V.P.; Ribeiro, M.N.G.; Molion, L.C.B.; Sá, L.D.A.; Nobre, C.A.; Cabral, O.M.R.; Patel, S.R.; Moraes, J.C. Observations of radiation exchange above and below Amazonian forest. Quarterly Journal of the Royal Meteorological Society, London, v.97, p.541-564, 1971.

Stewart, J.B. The albedo of a pine forest. Quarterly Journal of the Royal Meteorological Society, London, v.110, n.466, p.1163-1169, 1984.

Viswanadham, Y. Studies on radiation balance at a tropical station. Pure Applied Geophysics, London, v. 97, p.183-213, 1972. 